

\title{
Distributed control of systems over discrete groups
}

\author{
Benjamin Recht, Member, IEEE, Raffaello D'Andrea, Senior Member, IEEE,
}

\begin{abstract}
This paper discusses distributed controller design and analysis for distributed systems with arbitrary discrete symmetry groups. We show how recent results for designing controllers for spatially interconnected systems, based on semidefinite programming, are applicable to a much larger class of interconnection topologies. We also show how to exploit the structure of the symmetry group to produce a hierarchy of decreasingly conservative analysis and synthesis conditions.
\end{abstract}

Index Terms-Distributed control, H-infinity, interconnected systems, linear matrix inequalities

\section{INTRODUCTION}

With the advent of cheap sensors and pervasive communication and computing, there has been substantial activity in the controls community to develop analysis and synthesis tools for systems consisting of extremely large numbers of interconnected subsystems. A large part of this effort has been devoted to developing tools that scale gracefully with the number of subsystems, which in practice can each have local sensing, actuating, and computing elements. Clearly for systems that are comprised of a large number of subsystems (see [1], for example, for a description of a system which consists of thousands of interacting elements), the structure of these systems must be fully exploited in order to obtain tractable analysis and control synthesis algorithms.

Recent work has made a great deal of progress in exploiting the symmetry present in such systems. Control laws can be distributed such that they only rely on local communication, yet can still give rise to desired global behavior, and, in certain settings, it has been shown that spatially distributed controllers are optimal for the control of spatially invariant systems [2] [3]. The synthesis of such distributed controllers is often convex [4] [5], and taking the distributed structure of a problem into account can greatly reduce the complexity of control design without sacrificing system performance [6].

To date, most authors investigating distributed or decentralized control have focused on systems distributed over abelian groups. Finite difference approximations of partial differential equations on $\mathbb{R}^{n}$ or many systems connected on an integer lattice would fall into this category. However there are many spatially invariant configurations such as those arising from crystalline structures which have noncommutative symmetry groups. An investigation into how to exploit this symmetry in a distributed manner would open up a large new class of control systems for design.

In this paper, we show that recently presented techniques for the control design of spatially interconnected systems [7], [8], [9] are in fact applicable to a much larger class

This work was supported in part by the Center for Bits and Atoms (NSF CCR-0122419), by ARDA grant F30602-03-2-0090, by AFOSR grant F49620-98-1-0416, and by NSF grant ECS-9983954. of interconnection topologies where the symmetry of the interconnection may be noncommutative. We review these techniques in Section III, and in Section IV generalize the notion of spatial interconnectivity from abelian groups to arbitrary discrete groups. In Section $\mathrm{V}$ we discuss a linear matrix inequality (LMI) which can be used to analyze these more general systems and discuss how to use such an LMI for controller synthesis. In contrast to most existing techniques, the synthesis and analysis conditions are computationally tractable and always lead to a distributed controller implementation. Finally, in Section VI, we discuss how to make the LMI tests less conservative by using the structure of groups on which the signals are defined.

\section{NOTATION AND PRELIMINARIES}

$\mathbb{S}$ will denote an arbitrary discrete group. Unless otherwise noted, the identity element of $\mathbb{S}$ will be denoted by $\mathbf{1}$ and the group operation will be written as a product. We will be dealing with signals that are a function of both time and space. Elements of $\mathbb{S}$ will be used to denote the spatial index; in particular, signals are vector valued functions on $\mathbb{R} \times \mathbb{S}$. Formally, we define $l_{2}$ to be the Hilbert space of all functions $x: \mathbb{S} \rightarrow \mathbb{R}^{\bullet}$ such that the quantity

$$
\|x\|_{l_{2}}^{2}:=\sum_{\mathbf{s} \in \mathbb{S}} x(\mathbf{s})^{*} x(\mathbf{s})
$$

is finite. The Hilbert space $\mathcal{L}_{2}$ will denote the space of functions $u: \mathbb{R}^{+} \rightarrow l_{2}$ such that

$$
\|u\|_{\mathcal{L}_{2}}^{2}:=\int_{0}^{\infty}\|u(t)\|_{l_{2}}^{2} d t
$$

is finite.

With a slight abuse of notation, a signal $u \in \mathcal{L}_{2}$ can be considered a function of two independent variables $u=$ $u(t, \mathbf{s})$. For fixed $t$ and $\mathbf{s}, u(t)$ is an element of $l_{2}$ and $u(t, \mathbf{s})$ is a real-valued vector.

\section{ReView of Spatially Interconnected Systems}

In this section we give a brief review of the theory of spatially interconnected systems as presented in [8]. A "basic building block" (shown in Figure 1) for a spatially interconnected system is a linear time invariant system on an $L$ dimensional integer lattice defined as

$$
\left[\begin{array}{c}
\dot{x}(t, \mathbf{s}) \\
w(t, \mathbf{s}) \\
z(t, \mathbf{s})
\end{array}\right]=\left[\begin{array}{ccc}
A_{\mathrm{TT}} & A_{\mathrm{TS}} & B_{\mathrm{T}} \\
A_{\mathrm{ST}} & A_{\mathrm{Ss}} & B_{\mathbf{S}} \\
C_{\mathrm{T}} & C_{\mathbf{S}} & D
\end{array}\right]\left[\begin{array}{c}
x(t, \mathbf{s}) \\
v(t, \mathbf{s}) \\
d(t, \mathbf{s})
\end{array}\right]
$$


where $x(t=0) \in \ell_{2}$,

$$
v(t, \mathbf{s})=\left[\begin{array}{c}
v_{+1}(t, \mathbf{s}) \\
v_{-1}(t, \mathbf{s}) \\
\vdots \\
v_{+L}(t, \mathbf{s}) \\
v_{-L}(t, \mathbf{s})
\end{array}\right], \quad w(t, \mathbf{s})=\left[\begin{array}{c}
w_{+1}(t, \mathbf{s}) \\
w_{-1}(t, \mathbf{s}) \\
\vdots \\
w_{+L}(t, \mathbf{s}) \\
w_{-L}(t, \mathbf{s})
\end{array}\right],
$$

and $\mathbf{s}=\left(p_{1}, \ldots, p_{L}\right)$ is a fixed $L$-tuple of integers used to denote the position of the subsystem on the lattice. The vectors $v_{+}(t, \mathbf{s})$ and $w_{+}(t, \mathbf{s})$ are the same size, and $v_{-}(t, \mathbf{s})$ and $w_{-}(t, \mathbf{s})$ are the same size.

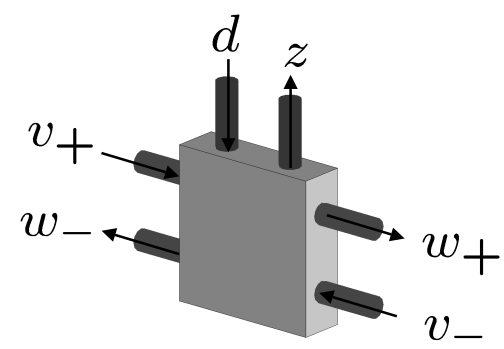

Fig. 1. A basic building block in one spatial dimension.

On an infinite extent integer lattice, the interconnection of these subsystems can be captured as follows. Define the shift operators $\mathbf{S}_{1}, \ldots, \mathbf{S}_{L}$ where for an arbitrary $x \in \ell_{2}$,

$$
\left(\mathbf{S}_{k} x\right)(\mathbf{s}):=x\left(p_{1}, \ldots, p_{k}+1, \ldots, p_{L}\right) .
$$

Periodicity of order $N$ on any axis can be imposed by defining the shift operator as follows

$$
\left(\mathbf{S}_{k} x\right)(\mathbf{s}):=x\left(p_{1}, \ldots,\left(p_{k}+1\right) \bmod N, \ldots, p_{L}\right) .
$$

We can extend these shift operators to $u \in \mathcal{L}_{2}$ in the following manner

$$
\left(\mathbf{S}_{k} u\right)(t):=\mathbf{S}_{k} u(t) .
$$

Let the dimensions of $v_{+k}$ and $v_{-k}$ be denoted by $m_{k}$ and $m_{-k}$ respectively and define the structured operator

$$
\boldsymbol{\Delta}=\operatorname{diag}\left(\mathbf{S}_{1} I_{m_{1}}, \mathbf{S}_{1}^{-1} I_{m_{-1}}, \ldots, \mathbf{S}_{L} I_{m_{L}}, \mathbf{S}_{L}^{-1} I_{m_{-L}}\right) .
$$

The interconnection of the subsystems is then simply defined to be $w=\boldsymbol{\Delta} v$. We can form an interconnected system as

$$
\left[\begin{array}{c}
\dot{x}(t, \mathbf{s}) \\
(\boldsymbol{\Delta} v)(t, \mathbf{s}) \\
z(t, \mathbf{s})
\end{array}\right]=\left[\begin{array}{ccc}
A_{\mathrm{TT}} & A_{\mathrm{TS}} & B_{\mathrm{T}} \\
A_{\mathrm{ST}} & A_{\mathrm{Ss}} & B_{\mathbf{s}} \\
C_{\mathrm{T}} & C_{\mathrm{S}} & D
\end{array}\right]\left[\begin{array}{c}
x(t, \mathbf{s}) \\
v(t, \mathbf{s}) \\
d(t, \mathbf{s})
\end{array}\right],
$$

$x(t=0) \in \ell_{2}$. Examples of such interconnections in one spatial dimension are shown in Figure 2. For clarity, such pictures will be simplified by lumping together the signals that interconnect two subsystems and by omitting the signals $d$ and $z$. as shown in Figure 3 .

There are three properties desired of such a system.

- Well-posedness: Well-posedness describes the realizability of the interconnection. An interconnected system defined by Equation (9) is well-posed if the operator $\left(\Delta-A_{\mathrm{ss}}\right)$ is invertible. The reader is referred to [8] for an in-depth discussion of well-posedness. Our definition

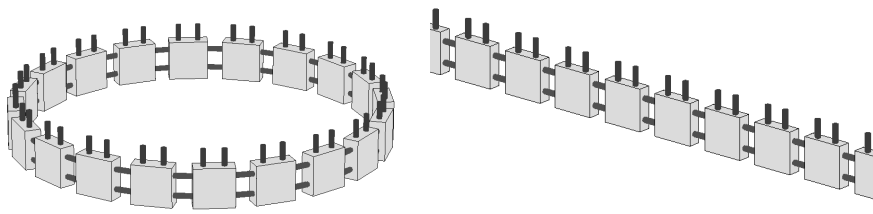

Fig. 2. Periodic and infinite one-dimensional interconnections.

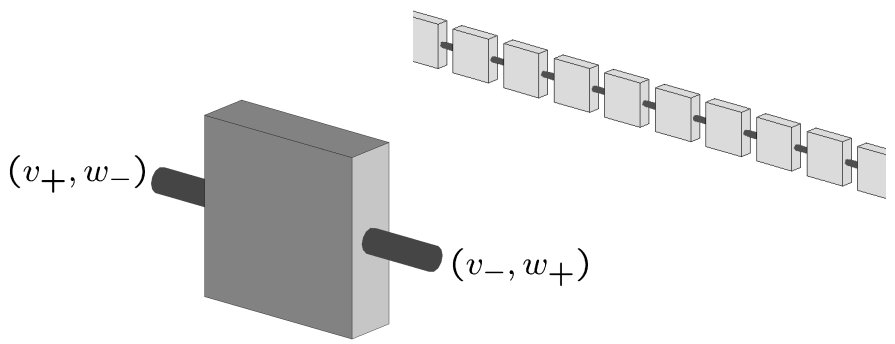

Fig. 3. Infinite interconnection in one spatial dimension with signals that interconnect two subsystems suppressed and the signals $d$ and $z$ omitted.

reflects the standard notion used for feedback interconnection; see [10], for example.

- Stability: A system is stable if, for any initial state $x(t=0)$, the norm of the signal $x$ is bounded above by a decaying exponential $\alpha \exp (-\beta t)$ when the input $d=0$.

- Contractiveness: A system is contractive if for any input signal $d \neq 0,\|z\|_{\mathcal{L}_{2}}<\|d\|_{\mathcal{L}_{2}}$ when $x(t=0)=0$.

The authors in [8] construct an LMI test which verifies well-posedness, stability, and contractiveness, and is only a function of the transition matrix of Equation (9). In particular, the resulting LMI is finite dimensional and fixed in size; it does not depend on the number of subsystems that make up the interconnection. The authors also describe how to use this LMI to synthesize distributed controllers, and these analysis and synthesis results have been extended to handle certain types of boundary conditions in [11]. The remainder of this paper is devoted to generalizing all of these results to a much richer class of interconnection topologies.

\section{Generalized Spatial Interconnections}

One approach to generalizing beyond the integer lattice structures considered thus far is to relax our notion of a shift operator. If we consider the basic building block in three dimensions, we can connect this together to form a cubic integer lattice as in Figure 4. We can also rearrange the signals in this basic building block and connect them as triangular lattice as in Figure 5. Similarly, we can create a hexagonal lattice as in Figure 6. In both of these new cases, there is still a well defined notion of a spatial shift, but the interconnection variables can no longer be broken down into $L$-tuples of integers. Instead, the variables will be indexed by elements of a discrete group.

Formally, let $\mathbb{S}$ be a group. The set of elements $G=$ $\left\{\mathbf{s}_{1}, \ldots, \mathbf{s}_{L}\right\}$ generates $\mathbb{S}$ if every element of $\mathbb{S}$ can be written as a product of elements from $G$ and inverses of elements from $G$. The elements $\mathbf{s}_{k}$ are called generators of $\mathbb{S}$. If $\mathbb{S}$ has 


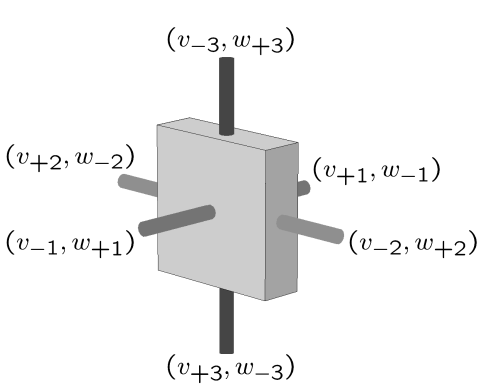

Fig. 4. Cubic integer lattice. The interconnection shown is a subsection of an infinite interconnection.

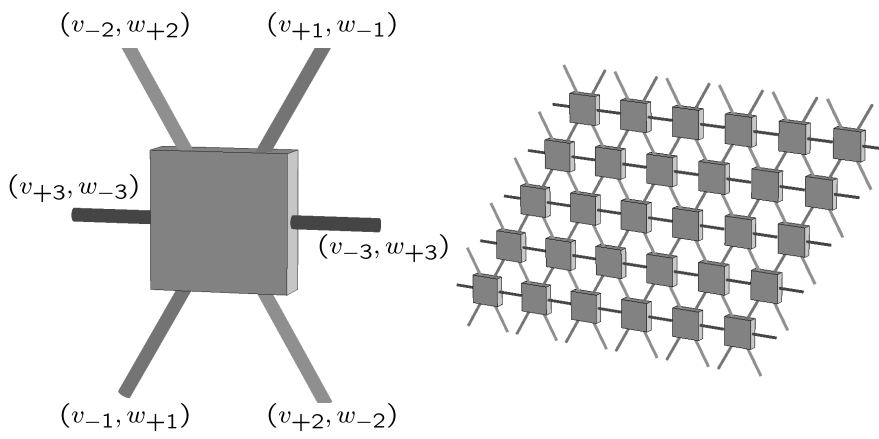

Fig. 5. Triangular lattice. The interconnection shown is a subsection of an infinite interconnection.

a finite generating set then it is finitely generated. The integer lattices in Section III are special cases of such finite generated groups. For example, in the case of a two-dimensional integer lattice, the group elements are given by locations on the lattice $\mathbf{s}=\left(p_{1}, p_{2}\right)$ and the group operation is componentwise addition. The group is generated by the two elements $\mathbf{s}_{1}=(1,0)$ and $\mathbf{s}_{2}=(0,1)$, and we have identities such as $\mathbf{s s}_{1}=\left(p_{1}+1, p_{2}\right)$ and $\mathbf{s}_{1}^{-1}=(-1,0)$.

The cubic, triangular, and hexagonal lattices are all generated by three elements. However, the generators relate to one another differently in each group. In the case of the cubic integer lattice, we can express the commutativity of the shift operators as

$$
\mathbf{s}_{1} \mathbf{s}_{2} \mathbf{s}_{1}^{-1} \mathbf{s}_{2}^{-1}=\mathbf{1} \quad \mathbf{s}_{2} \mathbf{s}_{3} \mathbf{s}_{2}^{-1} \mathbf{s}_{3}^{-1}=\mathbf{1} \quad \mathbf{s}_{3} \mathbf{s}_{1} \mathbf{s}_{3}^{-1} \mathbf{s}_{1}^{-1}=\mathbf{1}
$$

In the case of the triangular lattice, we add the additional requirement that

$$
\mathbf{s}_{1} \mathbf{s}_{2} \mathbf{s}_{3}=\mathbf{s}_{3} \mathbf{s}_{2} \mathbf{s}_{1}=\mathbf{1}
$$

For the hexagonal lattice we have instead

$$
\mathbf{s}_{1}^{2}=\mathbf{s}_{2}^{2}=\mathbf{s}_{3}^{2}=\left(\mathbf{s}_{1} \mathbf{s}_{2} \mathbf{s}_{3}\right)^{2}=\mathbf{1}
$$

Such products of elements which equal the identity are called relations. A group is finitely presented if there exist a set of generators $G$ and set of relations $R$ composed from the generators such that any relation for $\mathbb{S}$ can be written as a product of relations in $R$ or their inverses.

From any finitely presented group, we can create a directed graph as follows. The elements of $\mathbb{S}$ are the vertices. There is

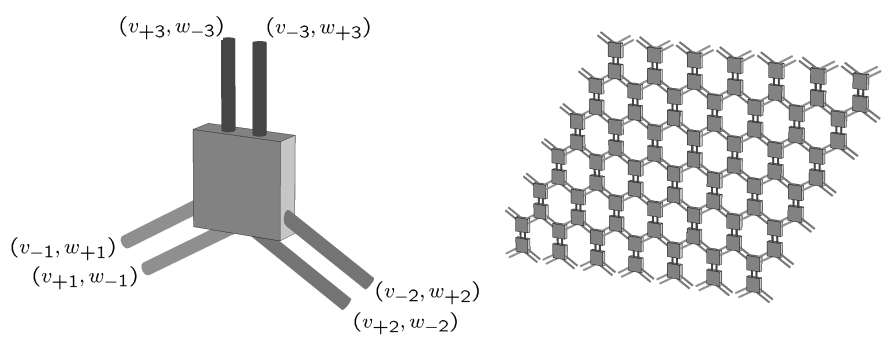

Fig. 6. Hexagonal lattice. The interconnection shown is a subsection of an infinite interconnection.

a directed edge from $\mathbf{a}$ to $\mathbf{b}$ if, for some $1 \leq k \leq L$, either $\mathbf{b}=\mathbf{a s}_{k}$ or $\mathbf{b}=\mathbf{a s}_{k}^{-1}$ where $\mathbf{s}_{k}$ is a generator. The resulting graph is called a Cayley graph [12].

We can define a spatially invariant system over any Cayley graph. Given a generator $\mathbf{s}_{k} \in G$ and $x \in l_{2}$, define the operator $\mathbf{S}_{k}$ by

$$
\left(\mathbf{S}_{k} x\right)(\mathbf{s}):=x\left(\mathbf{s s}_{k}\right) .
$$

Each of these shift operators on $l_{2}$ can be naturally extended to an operator on $\mathcal{L}_{2}$ as described in Section III. Examples of these shift operators are shown in Figure 7. From the perspective of the Cayley graph, these operators are unitary spatial-shifts. Accordingly, these shifts will play the role of the shift operators in Section III.

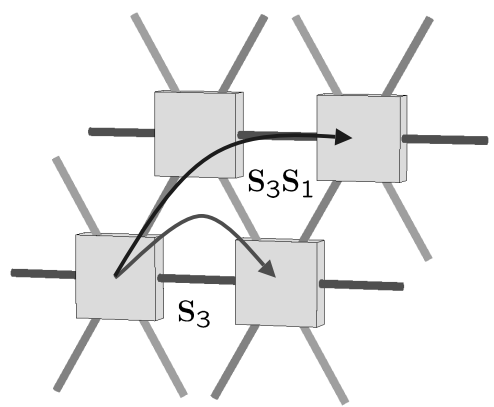

Fig. 7. Examples of shift operators on the triangular lattice.

To extend the results on interconnected systems to this more general setting, we will consider systems built from the same transition matrix as in the previous section, but we will now build shift operators from elements of $G$. Specifically, if $\mathbf{m}$ is the vector of dimensions of the interconnection signals $v$ in Equation (3) we can define a shift operator

$$
\boldsymbol{\Delta}:=\operatorname{diag}\left(\mathbf{S}_{1} I_{m_{1}}, \mathbf{S}_{1}^{-1} I_{m_{-1}}, \ldots, \mathbf{S}_{L} I_{m_{L}} \mathbf{S}_{L}^{-1} I_{m_{-L}}\right) .
$$

Now we can define the linear system over $\mathbb{S}$

$$
\left[\begin{array}{c}
\dot{x}(t, \mathbf{s}) \\
(\boldsymbol{\Delta} v)(t, \mathbf{s}) \\
z(t, \mathbf{s})
\end{array}\right]=\left[\begin{array}{ccc}
A_{\mathrm{TT}} & A_{\mathrm{TS}} & B_{\mathrm{T}} \\
A_{\mathrm{ST}} & A_{\mathrm{ss}} & B_{\mathrm{s}} \\
C_{\mathrm{T}} & C_{\mathrm{s}} & D
\end{array}\right]\left[\begin{array}{c}
x(t, \mathbf{s}) \\
v(t, \mathbf{s}) \\
d(t, \mathbf{s})
\end{array}\right],
$$

$x(t=0) \in \ell_{2}$.

Note again that the systems in Section III were the special case where the group $\mathbb{S}$ was a product of $L$ groups isomorphic to either the integers or the integers modulo $N$. The hexagonal lattice is an example of a noncommutative group. The ability to deal with spatial invariance over noncommutative symmetry 
groups is a new and crucial contribution of this work. The hexagonal lattice also has the interesting property that the generators square to $\mathbf{1}$; this relation can be used to identify the signals $v_{+k}$ with $v_{-k}$ and $w_{+k}$ with $w_{-k}$. The lattices we have presented are examples of two-dimensional space groups studied in abstract crystallography. The group which generates the triangular lattice is commonly called $\mathbf{p} 1$. The hexagonal group is called $\mathbf{p 2}$. There are 17 different space groups in 2D [13], and 230 in 3D [14]. The work in the sequel applies to all of them.

\section{LinEAR MATRIX INEQUALITIES FOR ANALYSIS AND CONTROLLER SYNTHESIS}

In this section, we discuss how the techniques in [8] can be immediately extended to systems on arbitrary discrete groups. We will provide a test for well-posedness, stability, and performance using only the data from Equation (15). It is worth noting that little changes in moving from systems defined over integer lattices to our more general situation. We can partition the matrices which govern the evolution of the system to reflect the structure of $\Delta$ :

$$
\begin{aligned}
& A_{\mathrm{ss}}=:\left[\begin{array}{cccc}
A_{\mathbf{s s}_{1,1}} & A_{\mathbf{s s}_{1,-1}} & \cdots & A_{\mathbf{s s}_{1,-}} \\
A_{\mathbf{s s}_{-1,1}} & A_{\mathbf{s s}_{-1,-1}} & \cdots & A_{\mathbf{s s}_{-1,-}} \\
& & \ddots & \\
A_{\mathbf{s s}_{-L, 1}} & A_{\mathbf{s s}_{-1,-1}} & \cdots & A_{\mathbf{s s}_{-L,-}}
\end{array}\right] \text {, } \\
& A_{\mathrm{ST}}=:\left[\begin{array}{c}
A_{\mathrm{ST}_{1}} \\
A_{\mathrm{ST}_{-1}} \\
\vdots \\
A_{\mathrm{ST}_{-L}}
\end{array}\right], \quad B_{\mathrm{S}}=:\left[\begin{array}{c}
B_{\mathrm{S}_{1}} \\
B_{\mathrm{S}_{-1}} \\
\vdots \\
B_{\mathrm{S}_{-L}}
\end{array}\right] \text {, } \\
& A_{\mathrm{TS}}=:\left[\begin{array}{llll}
A_{\mathrm{TS}_{1}} & A_{\mathrm{TS}_{-1}} & \cdots & A_{\mathrm{Ts}_{-L}}
\end{array}\right] \text {, } \\
& C_{\mathrm{s}}=:\left[\begin{array}{llll}
C_{\mathrm{s}_{1}} & C_{\mathrm{s}_{-1}} & \cdots & C_{\mathrm{s}_{-L}}
\end{array}\right],
\end{aligned}
$$

and then define the following matrices:

$$
\begin{aligned}
& A_{\mathrm{ss}}^{+}:=\left[\begin{array}{ccccc}
A_{\mathrm{ss}_{1,1}} & A_{\mathrm{ss}_{1,-1}} & \cdots & A_{\mathrm{ss}_{1, L}} & A_{\mathrm{ss}_{1,-L}} \\
0 & I & \cdots & 0 & 0 \\
& & \ddots & & \\
A_{\mathrm{ss}_{L, 1}} & A_{\mathrm{ss}_{L,-1}} & \cdots & A_{\mathrm{ss}_{L, L}} & A_{\mathrm{ss}_{L,-L}} \\
0 & 0 & \cdots & 0 & I
\end{array}\right] \text {, } \\
& A_{\mathrm{ST}}^{+}:=\left[\begin{array}{c}
A_{\mathbf{S T}_{1}} \\
0 \\
\vdots \\
A_{\mathrm{ST}_{L}} \\
0
\end{array}\right], B_{\mathrm{s}}^{+}:=\left[\begin{array}{c}
B_{\mathrm{s}_{1}} \\
0 \\
\vdots \\
B_{\mathrm{s}_{L}} \\
0
\end{array}\right] \\
& A_{\mathrm{ss}}^{-}:=\left[\begin{array}{ccccc}
I & 0 & \cdots & 0 & 0 \\
A_{\mathrm{ss}_{-1,1}} & A_{\mathrm{ss}_{-1,-1}} & \cdots & A_{\mathbf{s s}_{-1, L}} & A_{\mathrm{ss}_{-1,-}} \\
& & \ddots & & \\
0 & 0 & \cdots & I & 0 \\
A_{\mathbf{s s}_{-L, 1}} & A_{\mathrm{ss}_{-L,-1}} & \cdots & A_{\mathrm{ss}_{-L, L}} & A_{\mathbf{s s}_{-L}-L}
\end{array}\right],
\end{aligned}
$$

$$
\begin{aligned}
A_{\mathrm{ST}}^{-} & :=\left[\begin{array}{c}
0 \\
A_{\mathrm{ST}_{-1}} \\
\vdots \\
0 \\
A_{\mathrm{ST}_{-L}}
\end{array}\right], B_{\mathrm{S}}^{-}:=\left[\begin{array}{c}
0 \\
B_{\mathrm{S}_{-1}} \\
\vdots \\
0 \\
B_{\mathrm{S}_{-L}}
\end{array}\right], \\
A_{\mathrm{TS}}^{+} & :=\left[\begin{array}{lllll}
A_{\mathrm{TS}_{1}} & 0 & \cdots & A_{\mathrm{Ts}_{L}} & 0
\end{array}\right], \\
A_{\mathrm{TS}}^{-} & :=\left[\begin{array}{lllll}
0 & A_{\mathrm{TS}_{-1}} & \cdots & 0 & A_{\mathrm{Ts}_{-L}}
\end{array}\right] .
\end{aligned}
$$

Let $m_{0}$ denote the dimension of $x(t, \mathbf{s})$. Define the following sets of scaling matrices:

$$
\begin{gathered}
\mathcal{X}_{\mathrm{T}}:=\left\{X_{\mathrm{T}} \in \mathbb{R}^{m_{0} \times m_{0}}: X_{\mathrm{T}}>0, X_{\mathrm{T}}=X_{\mathrm{T}}^{*}\right\}, \\
\mathcal{X}_{\mathbf{S}}:=\left\{X_{\mathbf{S}}=\operatorname{diag}\left(X_{\mathbf{S}_{1}}, \cdots, X_{\mathbf{S}_{L}}\right):\right. \\
\left.X_{\mathbf{s}_{i}} \in \mathbb{R}^{\left(m_{i}+m_{-i}\right) \times\left(m_{i}+m_{-i}\right)}, X_{\mathbf{s}_{i}}=X_{\mathbf{s}_{i}}^{*}\right\} .
\end{gathered}
$$

The following result allows us to check the well-posedness, stability, and performance of the system defined by Equation (15) via an LMI which can be solved efficiently using semidefinite programming (see, for example [15]).

Theorem 1: A system defined by Equation (15) is wellposed, stable, and contractive if there exist $X_{\mathrm{T}}$ in $\mathcal{X}_{\mathrm{T}}$ and $X_{\mathrm{S}}$ in $\mathcal{X}_{\mathrm{s}}$ such that $J<0$, where

$$
\begin{aligned}
& J:=\left[\begin{array}{ccc}
I & 0 & 0 \\
A_{\mathrm{ST}}^{-} & A_{\mathrm{sS}}^{-} & B_{\mathrm{s}}^{-} \\
0 & 0 & I
\end{array}\right]^{*} \\
& \times\left[\begin{array}{ccc}
A_{\mathrm{TT}}^{*} X_{\mathrm{T}}+X_{\mathrm{T}} A_{\mathrm{TT}} & X_{\mathrm{T}} A_{\mathrm{TS}}^{+} & X_{\mathrm{T}} B_{\mathrm{T}} \\
\left(A_{\mathrm{TS}}^{+}\right)^{*} X_{\mathrm{T}} & -X_{\mathrm{S}} & 0 \\
B_{\mathrm{T}}^{*} X_{\mathrm{T}} & 0 & -I
\end{array}\right] \\
& \times\left[\begin{array}{ccc}
I & 0 & 0 \\
A_{\mathrm{ST}}^{-} & A_{\mathrm{sS}}^{-} & B_{\mathrm{s}}^{-} \\
0 & 0 & I
\end{array}\right] \\
& +\left[\begin{array}{ccc}
I & 0 & 0 \\
A_{\mathrm{sT}}^{+} & A_{\mathrm{ss}}^{+} & B_{\mathrm{s}}^{+} \\
C_{\mathrm{T}} & C_{\mathrm{s}} & D
\end{array}\right]^{*} \\
& \times\left[\begin{array}{ccc}
0 & X_{\mathrm{T}} A_{\mathrm{TS}}^{-} & 0 \\
\left(A_{\mathrm{TS}}^{-}\right)^{*} X_{\mathrm{T}} & X_{\mathrm{S}} & 0 \\
0 & 0 & I
\end{array}\right] \\
& \times\left[\begin{array}{ccc}
I & 0 & 0 \\
A_{\mathrm{sT}}^{+} & A_{\mathrm{sS}}^{+} & B_{\mathrm{s}}^{+} \\
C_{\mathrm{T}} & C_{\mathrm{S}} & D
\end{array}\right] .
\end{aligned}
$$

This analysis condition is identical to the one presented in [8] for systems defined over integer lattices. The LMI test is valid for both interconnections characterized by the structured operator of Equation (8) or the more general structured operator of Equation (14). Indeed, it is a sufficient condition for any interconnection with $L$ generators. The proof for the general interconnected system is also identical to the proof over integer lattices, so we refer the reader to [8] for the details. The essential ingredients for this LMI to be sufficient is for the operator $\boldsymbol{\Delta}$ to be unitary and commute with the transition matrices. This remains true in our more general setting, and hence the analysis condition still holds.

By defining our system in this more general fashion, we extend the possibility of studying controller design on a much larger class of distributed systems. For control design, we 
augment the basic building block with controller input/output variables $u$ and $y$ as

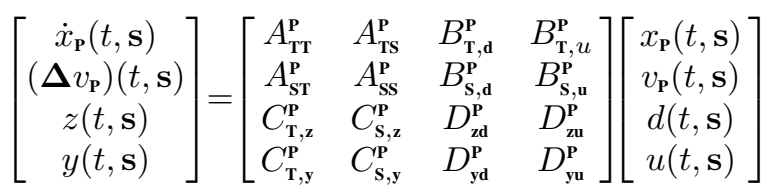

and aim to design a controller with the same structure as the plant, so we will posit the controller form of

$$
\left[\begin{array}{c}
\dot{x}_{\mathbf{K}}(t, \mathbf{s}) \\
\left(\boldsymbol{\Delta} v_{\mathbf{K}}\right)(t, \mathbf{s}) \\
u(t, \mathbf{s})
\end{array}\right]=\left[\begin{array}{ccc}
A_{\mathrm{TT}}^{\mathrm{K}} & A_{\mathrm{TS}}^{\mathrm{K}} & B_{\mathrm{T}}^{\mathbf{K}} \\
A_{\mathrm{ST}}^{\mathrm{K}} & A_{\mathrm{SS}}^{\mathrm{K}} & B_{\mathrm{s}}^{\mathbf{K}} \\
C_{\mathbf{T}}^{\mathbf{K}} & C_{\mathbf{s}}^{\mathbf{K}} & D^{\mathbf{K}}
\end{array}\right]\left[\begin{array}{c}
x_{\mathbf{K}}(t, \mathbf{s}) \\
v_{\mathbf{K}}(t, \mathbf{s}) \\
y(t, \mathbf{s})
\end{array}\right] .
$$

Here the superscripts and subscripts "P" and " $K$ " denote the plant and controller respectively. When we connect the signals $u$ and $y$ of the controller and the plant (see Figure 8) and eliminate these variables, we obtain the closed loop system of Equation (15). A calculation of the closed loop transition matrices is an algebraic manipulation of the plant and controller matrices, and can be found in [8].

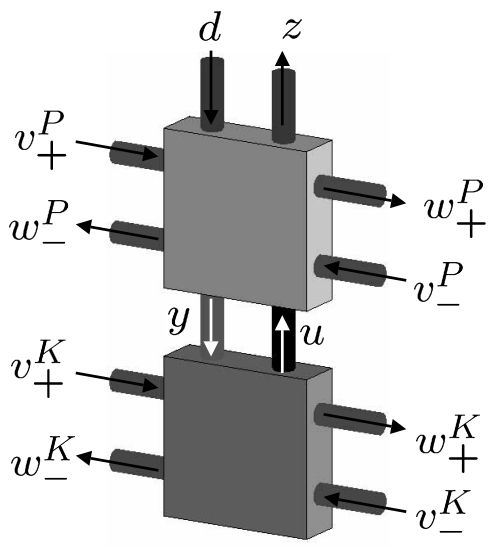

Fig. 8. The closed loop basic building block. The interconnection variables $v_{ \pm}^{P}, w_{p} m^{P}, v_{ \pm}^{K}$, and $w_{ \pm}^{K}$ are compressed into one dimension for visual clarity.

The controller synthesis problem thus consists of finding controller matrices - the matrices in Equation (30) - such that the closed loop system is well-posed, stable, and contractive. The case where there are no interconnection variables $v$ and $w$ reduces to the classic $H_{\infty}$ synthesis problem [16]. For the case of abelian groups, the analysis and synthesis problems have been studied in detail in [7], [8], [17], [2], and [5].

It is sufficient that the closed loop system satisfy the LMI in Theorem 1. Since this analysis condition is identical to what is obtained in [8] for systems on integer lattices, and the synthesis equations therein are based solely on this LMI, controller synthesis for the more general interconnection topologies considered in this paper can be performed using the same algorithms developed for integer lattices; the details are omitted. We note that the synthesis equations in [8], which take the form of LMIs, do not introduce additional conservatism in design; they are necessary and sufficient for a controller with the same structure as the plant to exist, as per Equation (30), such that the closed loop system satisfies the analysis LMI of Theorem 1.

\section{USING RELATIONS TO GENERATE LESS CONSERVATIVE LINEAR MATRIX INEQUALITIES}

Since the analysis LMI we have presented verifies wellposedness, stability, and contractiveness for all interconnected systems with the same number of generators, we might expect such a condition to overly conservative. We will now explore how to create LMI tests which are less conservative by collecting building blocks into subsystems and exploiting the Cayley graph structure to generate new conditions.

In order to simplify the presentation, some of the terminology we use is not standard. We will restrict our attention to a particular class of of subgroups of $\mathbb{S}$.

Definition 1: We say that a subset $\mathbb{H}$ of $\mathbb{S}$ is a central subgroup if

- $\mathbb{H}$ is a group under the group operation of $\mathbb{S}$.

- $\mathbb{H}$ is finitely presented.

- $\mathbf{s h}=\mathbf{h s}$ for all $\mathbf{s} \in \mathbb{S}$ and $\mathbf{h} \in \mathbb{H}$.

- Under the identification $\mathbf{a} \sim \mathbf{b}$ (that is, $\mathbf{a}$ is equivalent to b) if $\mathbf{a}=\mathbf{b h}$ for some $\mathbf{h} \in \mathbb{H}$, there are finitely many equivalence classes.

Denote the set of equivalence classes under $\mathbb{H}$ by $\mathbb{S} / \mathbb{H}$ and the map from $\mathbb{S}$ to $\mathbb{S} / \mathbb{H}$ by $\eta$. $\mathbb{S} / \mathbb{H}$ inherits a group structure from $\mathbb{S}$ by imposing the relations $\mathbf{h}_{k}=\mathbf{1}$ for each generator of $\mathbb{H}$. Since the generators for $\mathbb{S} / \mathbb{H}$ and $\mathbb{S}$ are the same, $\mathbb{S} / \mathbb{H}$ has a Cayley graph generated by the generators of $\mathbb{S}$. That is, the spatial-shift operators of the group $\mathbb{S}$ map to spatial-shift operators on $\mathbb{S} / \mathbb{H}$.

Definition 2: A transversal is a set $T=(V, E)$ of vertices $V$ and edges $E$ in the Cayley graph such that

- The map $\eta$ is a bijection when restricted to $V$.

- $E$ is the set of all edges beginning at a vertex in $T$.

- The identity element of $\mathbb{S}$ is contained in $V$.

- $T$ is a connected subgraph.

Transversals are liftings from the Cayley graph of $\mathbb{S} / \mathbb{H}$ back to the Cayley graph of $\mathbb{S}$ via the inverse of $\eta$ [18]. To make these definitions clear, consider again the two-dimensional integer lattice group and the subgroup $\mathbb{H}$ generated by even powers of $\mathbf{s}_{1}$ and $\mathbf{s}_{2}$. The quotient group $\mathbb{S} / \mathbb{H}$ is obtained by imposing the relations $\mathbf{s}_{1}^{2}=1$ and $\mathbf{s}_{2}^{2}=1$. It is then readily seen that $\mathbb{S} / \mathbb{H}$ is isomorphic to the finite group which is the product of two groups of each with two elements. We can lift this group back to the transversal $T$. $V$ consists of the elements $\mathbf{1}, \mathbf{s}_{1}, \mathbf{s}_{2}$, and $\mathbf{s}_{1} \mathbf{s}_{2}$. The transversal is graphically depicted in Figure 9.

We can generate the entire Cayley graph using translations only contained in $\mathbb{H}$ and the transversal $T$. Let $T \mathbf{h}$ denote the translation of the edges and vertices in the transversal by the element $\mathbf{h} \in \mathbb{H}$. Each node in the Cayley graph is contained in a unique translation, since if $\mathbf{s}$ is a node in $V$, then $\mathbf{s h}=$ $\mathbf{s h}^{\prime}$ if and only if $\mathbf{h}=\mathbf{h}^{\prime}$. Now suppose there is an edge from $\mathbf{a}$ to $\mathbf{b}$ in the Cayley graph of $\mathbb{S}$. Then $\mathbf{b}=\mathbf{a s}_{k}$ for one of the generators of $\mathbb{S}$. Since $\mathbb{H}$ is central, it follows that for every $\mathbf{h} \in H$, $\mathbf{b h}=\mathbf{a s}_{k} \mathbf{h}=\mathbf{a h s}_{k}$ and hence there is an edge from ah to bh in the Cayley graph. Therefore, any nodes which are connected to each other in the transversal are also connected in any translation of the transversal, and hence the entire graph can be constructed by translating the 


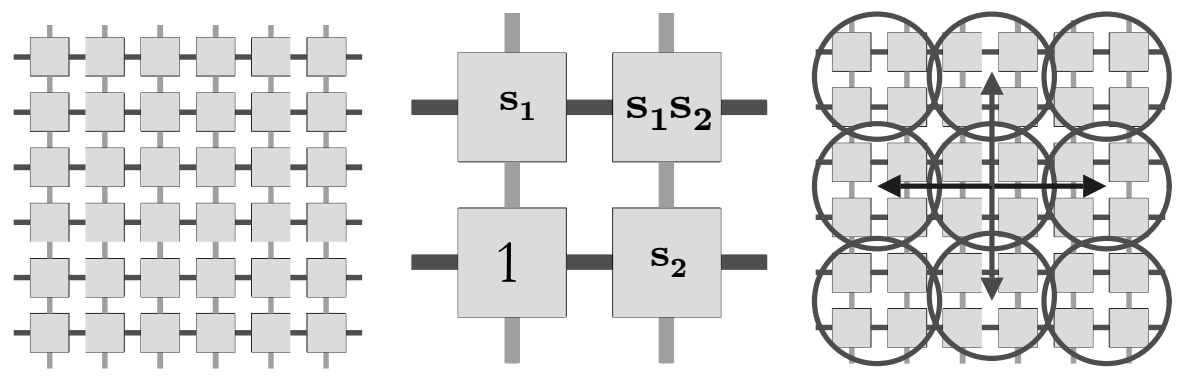

Fig. 9. An example of a transversal and its associated central subgroup. The transversal consists of four nodes. Translating the transversal by the subgroup generates the entire Cayley graph. The interconnection shown is a subsection of an infinite interconnection.

transversal by elements of $\mathbb{H}$. From this perspective, $\mathbb{H}$ serves to group nodes of the Cayley graph of $\mathbb{S}$ into clusters that form a Cayley graph for $\mathbb{H}$. Given an interconnected system defined by Equation (15), the signals associated with $T$ form a basic building block for an interconnection given by $\mathbb{H}$, and we can construct new conditions for well-posedness, stability, and contractiveness.

To make the clustering explicit, take the set of edges which connect nodes in the transversal to be interior edges. The set of all edges beginning at nodes in the transversal which are not interior edges are called exterior edges. Now consider the interconnected system defined by Equation (15). For each $\mathbf{h} \in$ $H$ we can stack all of the signals involving vertices in $T \mathbf{h}$ as follows. Stack the set of internal state variables in a vector

$$
\begin{aligned}
x(t, \mathbf{h})= & {\left[x(t, \mathbf{h}), x\left(t, \mathbf{g}_{1} \mathbf{h}\right), \ldots x\left(t, \mathbf{g}_{M} \mathbf{h}\right)\right], } \\
\mathbf{g}_{k} & \in V, \mathbf{h} \in \mathbb{H}
\end{aligned}
$$

and the disturbance and output signals can be stacked accordingly. Stack the interconnection signals corresponding to interior edges as $v_{\mathbf{I}}(t, \mathbf{h})$ and $w_{\mathbf{I}}(t, \mathbf{h})$ and those corresponding to exterior edges as $v_{\mathbf{E}}(t, \mathbf{h})$ and $w_{\mathbf{E}}(t, \mathbf{h})$. Each signal $w_{ \pm k}(t, \mathbf{s h})$ in $w_{\mathbf{I}}(t, \mathbf{h})$ corresponds to a signal $v_{\mp k}\left(t, \mathbf{s s}_{k}^{\mp 1} \mathbf{h}\right)$ in $v_{\mathbf{I}}(t, \mathbf{h})$. Similarly, each signal $w_{ \pm k}(t, \mathbf{s h})$ in $w_{\mathbf{E}}(t, \mathbf{h})$ corresponds to a signal $v_{\mp k}\left(t, \mathbf{s s}_{k}^{\mp 1} \mathbf{h}\right)$ in $v_{\mathbf{E}}(t, \mathbf{h})$. Thus, there exists two structured operators $\Pi$ and $\Theta$, similar to those in Equation (14), such that the system

$$
\left[\begin{array}{c}
\dot{x}(t, \mathbf{h}) \\
\left(\Pi v_{\mathrm{I}}\right)(t, \mathbf{h}) \\
\left(\Theta v_{\mathrm{E}}\right)(t, \mathbf{h}) \\
z(t, \mathbf{h})
\end{array}\right]=\left[\begin{array}{cccc}
A_{\mathrm{TT}} & A_{\mathrm{TI}} & A_{\mathrm{TE}} & B_{\mathrm{T}} \\
A_{\mathrm{IT}} & A_{\mathrm{II}} & A_{\mathrm{IE}} & B_{\mathrm{I}} \\
A_{\mathrm{ET}} & A_{\mathrm{EI}} & A_{\mathrm{EE}} & B_{\mathrm{E}} \\
C_{\mathrm{T}} & C_{\mathrm{I}} & C_{\mathrm{E}} & D
\end{array}\right]\left[\begin{array}{c}
x(t, \mathbf{h}) \\
v_{\mathrm{I}}(t, \mathbf{h}) \\
v_{\mathbf{E}}(t, \mathbf{h}) \\
d(t, \mathbf{h})
\end{array}\right]
$$

with $\mathbf{h} \in \mathbb{H}$ is identical to the system in Equation (15). Under this new identification, note that the operator $\Pi$ only permutes the elements of $v_{\mathbf{I}}(t, \mathbf{h})$. $\Pi$ does not couple the newly grouped subsystems. Hence, it can be treated as a permutation matrix of size compatible with the vector $v_{\mathbf{I}}(t, h)$. The following proposition gives a quick way to test the well-posedness of an interconnected system if some information about the subgroup structure of $\mathbb{S}$ is known.

Proposition 1: If the matrix $\left(\Pi-A_{\mathrm{II}}\right)$ is not invertible, then the system is not well-posed.

Proof: of Proposition 1 It is clear that if we replace the shifted signal $\left(\Theta v_{\mathbf{E}}\right)(t, \mathbf{h})$ in Equation (32) with a signal of compatible size $w_{\mathrm{E}}$, then if the system with the variable $w_{\mathrm{E}}$ is not well-posed, the interconnected system is also not wellposed. For a fixed $\mathbf{h}$, this system is a finite dimensional linear time-invariant system, and is well-posed if and only if $\left(\Pi-A_{\text {II }}\right)$ is invertible (c.f. [10]).

If $\left(\Pi-A_{\text {II }}\right)$ is invertible, we can proceed to generate an LMI for analysis by defining the matrices

$$
\begin{aligned}
{\left[\begin{array}{ccc}
\tilde{A}_{\mathrm{TT}} & \tilde{A}_{\mathrm{TS}} & \tilde{B}_{\mathrm{T}} \\
\tilde{A}_{\mathrm{ST}} & \tilde{A}_{\mathrm{SS}} & \tilde{B}_{\mathrm{S}} \\
\tilde{C}_{\mathrm{T}} & \tilde{C}_{S} & \tilde{D}
\end{array}\right] } & =\left[\begin{array}{ccc}
A_{\mathrm{TT}} & A_{\mathrm{TE}} & B_{\mathrm{T}} \\
A_{\mathrm{ET}} & A_{\mathrm{EE}} & B_{\mathrm{E}} \\
C_{\mathrm{T}} & C_{\mathrm{E}} & D
\end{array}\right] \\
& +\left[\begin{array}{c}
A_{\mathrm{TI}} \\
A_{\mathrm{EI}} \\
C_{\mathrm{I}}
\end{array}\right]\left(\Pi-A_{\mathrm{II}}\right)^{-1}\left[\begin{array}{lll}
A_{\mathrm{IT}} & A_{\mathrm{IE}} & B_{\mathrm{I}}
\end{array}\right]
\end{aligned}
$$

and eliminating the variables $v_{\mathrm{I}}$. This yields the equivalent formulation

$$
\left[\begin{array}{c}
\dot{x}(t, \mathbf{h}) \\
\left(\Theta v_{\mathrm{E}}\right)(t, \mathbf{h}) \\
z(t, \mathbf{h})
\end{array}\right]=\left[\begin{array}{ccc}
\tilde{A}_{\mathrm{TT}} & \tilde{A}_{\mathrm{TS}} & \tilde{B}_{\mathrm{T}} \\
\tilde{A}_{\mathrm{ST}} & \tilde{A}_{\mathrm{SS}} & \tilde{B}_{\mathrm{S}} \\
\tilde{C}_{\mathrm{T}} & \tilde{C}_{\mathrm{S}} & \tilde{D}
\end{array}\right]\left[\begin{array}{c}
x(t, \mathbf{h}) \\
v_{\mathrm{E}}(t, \mathbf{h}) \\
d(t, \mathbf{h})
\end{array}\right] .
$$

This new realization of our system can now be fed into the LMI tests of the previous section and new, potentially less conservative bounds on performance can be obtained.

The process of collecting nodes by normal subgroups may be repeated an arbitrary number of times when the group in question has infinite order. Once the subsystems are collected, normal subgroups of the new group structure can be used to repeat this analysis yielding a hierarchy of less conservative LMIs.

\section{CONCLUSIONS}

There are many interesting directions for further investigation. The grouping process of Section VI produces state-space matrices of increasingly larger size, and determining the tradeoff between conservatism and LMI complexity is an important consideration for analysis. Furthermore, we can use the results of Section V to generate hierarchical controllers connected to all sub-units which are clustered together. Examining how to scale this hierarchy is an interesting thread for future inquiry.

Other directions for future work include a careful analysis of how combining the same building block in different configurations affects stability and performance. It would also be interesting to find tests which explicitly exploit the group structure using noncommutative harmonic analysis in a manner 
similar to how the Locally Compact Abelian structure is exploited in [2] to produce necessary and sufficient frequency domain conditions. In this case, we might be able to extend our results to systems over continuous non-abelian groups such as Lie Groups. Finally, recent results in minimal realization theory for linear fractional transformations [19] show that LMI conditions for minimality are both necessary and sufficient when the operators describing the transformations are noncommutative. It would be very interesting to produce similar necessary conditions for the LMIs studied here by studying the noncommutative structure of group operators.

\section{ACKNOWLEDGMENT}

The authors would like to thank R. Chandra, C. Langbort, P. Parrilo, and the reviewers for their helpful comments and suggestions.

\section{REFERENCES}

[1] W. J. Butera, Programing a Paintable Computer. PhD thesis, Massachusetts Institute of Technology, Cambridge,MA, 2002.

[2] B. Bamieh, F. Paganini, and M. Dahleh, "Distributed control of spatially invariant systems," IEEE Transactions on Automatic Control, vol. 47, no. 7, pp. 1091-1118, 2002.

[3] N. C. Martins, S. Venkatesh, and M. A. Dahleh, "Controller design and implementation for large-scale systems, a block decoupling approach," in Proceedings of the American Control Conference, pp. 4728-4733, 2001.

[4] G. A. de Castro and F. Paganini, "Convex synthesis of localized controllers for spatially invariant systems," Automatica, vol. 38, pp. 445456, 2002.

[5] P. Voulgaris, G. Bianchini, and B. Bamieh, "Optimal decentralized controllers for spatially invariant systems," in Proceedings of the 39th IEEE Conference on Decision and Control, pp. 3763 -3768, 2000.

[6] R. Cogill, S. Lall, and P. A. Parrilo, "On structured semidefinite programs for the control of symmetric systems," in Proceedings of the Allerton Conference on Communication, Control, and Computing, 2003.

[7] R. D'Andrea, "A linear matrix inequality approach to decentralized control of distributed parameter systems," in Proceedings of the 36th Conference on Decision and Control, pp. 1350-1354, 1997.

[8] R. D'Andrea and G. E. Dullerud, "Distributed control design for spatially interconnected systems," IEEE Transactions on Automatic Control, vol. 48, no. 9, pp. 1478-1495, 2003.

[9] R. D'Andrea, C. Langbort, and R. Chandra, "A state space approach to control of interconnected systems," in Mathematical Systems Theory in Biology, Communication, Computation and Finance (J. Rosenthal, ed.), Springer, IMA Book Series, 2003. To appear.

[10] K. Zhou, J. C. Doyle, and K. Glover, Robust and Optimal Control. New Jersey: Prentice Hall, 1995.

[11] C. Langbort and R. D'Andrea, "Imposing boundary conditions for a class of spatially interconnected systems," in Proceedings of the 2003 American Control Conference, pp. 107-112, 2003.

[12] J. J. Rotman, An Introduction to the Theory of Groups. Berlin: SpringerVerlag, 1995.

[13] H. S. M. Coxeter and W. O. J. Moser, Generators and Relations for Discrete Groups. Berlin: Springer-Verlag, 1965.

[14] C. J. Bradley and A. P. Cracknell, The Mathematical Theory of Symmetry in Solids. Oxford: Clarendon Press, 1972.

[15] S. P. Boyd, L. E. Ghaoui, E. Feron, and V. Balakrishnan, Linear Matrix Inequalities in System and Control Theory. Philadelphia: SIAM studies in applied mathematics, 1994.

[16] J. C. Doyle, K. Glover, P. Khargonekar, and B. A. Francis, "State-space solutions to standard $\mathcal{H}_{2}$ and $\mathcal{H}_{\infty}$ control problems," IEEE Transactions on Automatic Control, vol. 34, no. 8, pp. 831-847, 1989.

[17] B. Bamieh, "The structure of optimal controllers of spatially-invariant distributed parameter systems," in Proceedings of the 36th Conference on Decision and Control, pp. 1056-1061, 1997.

[18] W. Dicks and M. J. Dunwoody, Groups acting on graphs. Cambridge, England: Cambridge University Press, 1989.

[19] C. Beck and J. C. Doyle, "A necessary and sufficient minimality condition for uncertain systems," IEEE Transactions on Automatic Control, vol. 44, pp. 1802-1813, 1999.

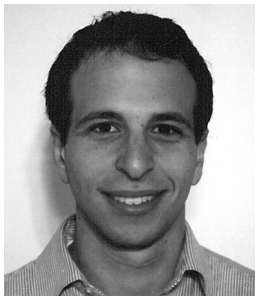

Benjmain Recht received a B.S. degree from the University of Chicago in 2000, and a M.S. degree from the Massachusetts Institute of Technology in 2002. He is currently a PhD candidate at the MIT Media Lab working in the NSF Center for Bits and Atoms.

He was the recipient of the Cohen Prize from the University of Chicago Mathematics Department and was awarded an MIT Presidential Fellowship. His electronic artwork has been exhibited at the ARCO festival in Madrid, Spain, the Museum of Contemporary Art in Barcelona, Spain and the ARS Electronica Center in Linz, Austria and received 3rd prize at the VIDA 6.0 Art and Artificial Life Competition in 2003.

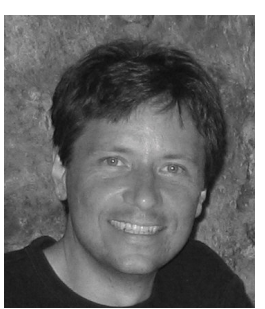

Raffaello D'Andrea was born in Pordenone, Italy, in 1967. He received the BASc. degree in Engineering Science from the University of Toronto in 1991, and the M.S. and Ph.D. degrees in Electrical Engineering from the California Institute of Technology in 1992 and 1997, respectively. Since then, he has been with the Department of Mechanical and Aerospace Engineering at Cornell University, where he is an Associate Professor. He is also Vice President of Systems Architecture for Distrobot Systems Incorporated.

He has been a recipient of the University of Toronto W.S. Wilson Meda in Engineering Science, an IEEE Conference on Decision and Control best student paper award, an American Control Council O.Hugo Schuck Best Paper award, an NSF CAREER Award, and a DOD sponsored Presidential Early Career Award for Scientists and Engineers. He was the system architect and faculty advisor of the RoboCup world champion Cornell Autonomous Robot Soccer team in 1999 (Stockholm, Sweden), 2000 (Melbourne, Australia), 2002 (Fukuoka, Japan), and 2003 (Padova, Italy), and third place winner in 2001 (Seattle, USA). His recent collaboration with Canadian artist Max Dean, "The Table", an interactive installation, appeared in the Biennale di Venezia in 2001. It was on display at the National Gallery of Canada in Ottawa, Ontario, in 2002 and 2003, and is now part of its permanent collection. 